\title{
A counterexample to a Penrose inequality conjectured by Gibbons
}

\author{
Sergio Dain ${ }^{1,2}$, Gilbert Weinstein ${ }^{3}$ and Sumio Yamada ${ }^{4}$ \\ ${ }^{1}$ Facultad de Matemática, Astronomía y Física, FaMAF, \\ Universidad Nacional de Córdoba, \\ Instituto de Física Enrique Gaviola, IFEG, CONICET, \\ Ciudad Universitaria, Argentina. \\ ${ }^{2}$ Max Planck Institute for Gravitational Physics, \\ (Albert Einstein Institute), Germany. \\ ${ }^{3}$ School of Mathematics, Monash University \\ ${ }^{4}$ Mathematical Institute \\ Tohoku University, Japan
}

July 7, 2018

\begin{abstract}
We show that the Brill-Lindquist initial data provides a counterexample to a Riemannian Penrose inequality with charge conjectured by G. Gibbons. The observation illustrates a sub-additive characteristic of the area radii for the individual connected components of an outermost horizon as a lower bound of the ADM mass.
\end{abstract}

\section{Introduction}

Let $\left(M, g_{0}\right)$ be an asymptotically flat three dimensional manifold, with nonnegative scalar curvature $R_{0}$. Given an asymptotically flat end, let us assume that there exists a set of minimal 2-spheres acting as the outermost horizon. In this situation, there are a series of inequalities which relate the asymptotic data with the Riemannian geometry of the manifolds.

The first such inequality is the Positive Mass Theorem 14, 15, 18. We rephrase the Riemannian version of this result as the following variational statement: among all time-symmetric asymptotically flat initial data sets for the Einstein-Vacuum Equations, flat Euclidean 3-space is the unique minimizer of the total mass. Thus, the total mass satisfies $m \geq 0$ with equality if and only if the data set is isometric to $\mathbb{R}^{3}$ with the flat metric.

A stronger result is the Riemannian version of the Penrose Inequality 12, 1, 8] (see also the review article [10] and references therein), which can be stated 
in a similar variational vein: among all time-symmetric asymptotically flat initial data sets for the Einstein-Vacuum Equations with an outermost minimal surface of area $A$, the Schwarzschild slice is the unique minimizer of the total mass. In other words, $m \geq R / 2$ where $R=\sqrt{A / 4 \pi}$ is the area radius of the outermost horizon, and equality occurs if and only if the data is isometric to the Schwarzschild slice:

$$
g_{i j}=\left(1+\frac{m}{2 r}\right)^{4} \delta_{i j} .
$$

When these results are phrased in this fashion, a natural question is whether similar variational characterizations of the other known stationary solutions of the Einstein equations hold. In particular, one could ask whether among all asymptotically flat Einstein-Maxwell initial data set with an horizon of area $A$ and charge $Q$ the Reissner-Nordström slice is the unique minimizer of the mass. This is equivalent to asking whether the following inequality holds for any data set:

$$
m \geq \frac{1}{2}\left(R+\frac{Q^{2}}{R}\right),
$$

where $Q$ is the total charge, with equality if and only if the data are a ReissnerNordström slice. The charge within a 2 -surface $S$ is defined by

$$
Q(S)=\frac{1}{4 \pi} \int_{S} E_{i} n^{i} d A
$$

It depends only on the homological type of $S$.

When the horizon is connected, inequality (11) can be proved by using the Inverse Mean Curvature flow [8, 9]. Indeed, the argument in [9] relies simply on Geroch monotonicity of the Hawking mass - which still holds for the weak flow introduced by Huisken and Ilmanen in 8 - while keeping track of the scalar curvature term $R=2\left(|E|^{2}+|B|^{2}\right)$. However, when the horizon has several components the same argument yields the following inequality:

$$
m \geq \frac{1}{2} \max _{i}\left(R_{i}+\frac{\left(\min \sum_{i} \varepsilon_{i} Q_{i}\right)^{2}}{R_{i}}\right),
$$

where $R_{i}$ and $Q_{i}$ are the area radii and charges of the components of the horizon $i=1, \ldots, N, \varepsilon_{i}=0$ or 1 , and the minimum is taken over all possible combinations.

In 17. we pointed out that (11) does not hold for the case of an horizon with several disconnected components. Namely, there exists a strongly asymptotically flat time-symmetric initial data set $(M, g, E, 0)$ for the Einstein-Maxwell Equations such that:

$$
m<\frac{1}{2}\left(R+\frac{Q^{2}}{R}\right) .
$$

In 1984, Gibbons [7 conjectured an inequality similar to (11). However, in his conjecture, the right hand side of (1) is taken to be additive over connected 
components of the horizon. Thus, Gibbons's conjecture states that:

$$
m \geq \frac{1}{2} \sum_{i}\left(R_{i}+\frac{Q_{i}^{2}}{R_{i}}\right) .
$$

There is a physical reason to introduce additive quantities on the right hand side of the inequality. The quantity

$$
m_{i}=\frac{1}{2}\left(R_{i}+\frac{Q_{i}^{2}}{R_{i}}\right),
$$

appears to play the role of the quasi-local mass of the $i$-th black holes. Then, inequality (5) can be interpreted as saying that the total mass of the spacetime is always bigger than the sum of the quasi-local masses of the individual black holes. There is, however, a Newtonian reasoning to doubt inequality (5) in the case of several black holes. When the black holes are separated by a large distance, it is expected that the interaction energy between them, which is asymptotically Newtonian in this limit, will be negative. The total energy of the spacetime should be the sum of the quasi-local masses of the black holes plus this negative interaction energy. Hence the sum of the quasi-local masses is expected to be bigger than the total mass. The counterexample we present in the next section exhibits precisely this behavior.

It is important to note that there is an inequality analogous to (5) for the Kerr black hole:

$$
m \geq \sum_{i} m_{i}
$$

where the quasi-local masses are now defined by:

$$
m_{i}=\sqrt{\frac{R_{i}^{2}}{4}+\frac{J_{i}^{2}}{R_{i}^{2}}},
$$

Here, $J_{i}$ denotes the angular momentum of the $i$-th black hole. Unlike the quasi-local charge (2), it is not straightforward to define the quasi-local angular momentum of each black holes in general; see the review article [16] on the problem of quasi-local mass and angular momentum. However, in the case of axial symmetry, there is a natural definition: the Komar integral. In that case, we can ask whether inequality (7) holds. Finally, we can also combine, using the Kerr-Newman black hole solution, both inequalities to obtain the general inequality with charge and angular momentum. Namely, define the $m_{i}$ to be

$$
m_{i}=\sqrt{\frac{1}{4}\left(R_{i}+\frac{Q_{i}^{2}}{R_{i}}\right)^{2}+\frac{J_{i}^{2}}{R_{i}^{2}}}
$$

While it is still an open problem, inequality (7) for one black hole in axial symmetry is expected to be hold; see the discussion in [10] and [5]. However, if we assume that there are two or more black holes, our counterexample is 
relevant since when we set the charges and the angular momentum to zero, all these inequalities imply:

$$
m \geq \frac{1}{2} \sum_{i} R_{i},
$$

which, our example violates. This inequaity is stronger than the usual Riemannian Penrose inequality [1]

$$
m \geq \frac{1}{2}\left(\sum_{i} R_{i}^{2}\right)^{1 / 2} .
$$

As mentioned above, and as pointed out also in 6, 17, the natural candidate to violate inequality (10) is a configuration of two Schwarzschild black holes separated by a large distance. This is precisely the counterexample we present. Although all the ingredients used in our argument have been present in the literature, it has not been pointed out before to the best of our knowledge. We believe that this counterexample is important because it sheds some light on the quasi-local aspect of Riemannian Penrose inequalities.

\section{Counterexample}

The Riemannian manifold that violates the inequality (10) proposed by Gibbons [7] is the well known Brill-Lindquist data [2, which is a conformally flat timesymmetric vacuum data defined on the differentiable manifold $M:=\mathbb{R}^{3} \backslash\left\{x_{1}, x_{2}\right\}$ with the metric $h_{i j}=\phi^{4} \delta_{i j}$ with the conformal factor

$$
\phi=\left(1+\frac{\mu_{1}}{2\left|x-x_{1}\right|}+\frac{\mu_{2}}{2\left|x-x_{2}\right|}\right) .
$$

"Time-symmetric" here means that the second fundamental form of the three manifold in the spacetime that is a solution to the Einstein equation vanishes. As $\phi$ is harmonic on $\mathbb{R}^{3}$, the scalar curvature of the metric is zero. For the sake of simplicity, we make the assumption $\mu_{1}=\mu_{2}=: \mu$, and $x_{1}=(0,0,1)$, $x_{2}=(0,0,-1)$.

The manifold $(M, h)$ has three asymptotically flat ends, namely $E_{0}$ where $\left|x-x_{1}\right|,\left|x-x_{2}\right| \rightarrow \infty, E_{1}$ where $x \rightarrow x_{1}$ and $E_{2}$ on which $x \rightarrow x_{2}$. The end $E_{0}$ has mass $m\left(E_{0}\right)=\mu_{1}+\mu_{2}=2 \mu$. Let $\Omega_{r}=\left\{\left|x-x_{1}\right|>1 / r,\left|x-x_{2}\right|>1 / r,|x|<\right.$ $r\}$ and let $S_{r, i}=\left\{\left|x-x_{i}\right|=1 / r\right\}$ for $i=1,2$, and $S_{0}=\{|x|=r\}$, then for $r$ large enough, the boundary $S_{r, 0} \cup S_{r, 1} \cup S_{r, 2}$ of $\Omega_{r}$ has positive mean curvature with respect to the outer normal. Hence, by minimizing area over all surfaces enclosing $S_{1}$ and $S_{2}$ and enclosed in $S_{0}$, one can show that there is a surface of least area $\Sigma$ enclosing $E_{1}$ and $E_{2}$, see [11, Theorem 1', p. 645]. Suppose that $\Sigma=\Sigma_{1} \cup \Sigma_{2}$ where $\Sigma_{i}$ is a compact minimal surface which encloses $E_{i}$, and $A\left(\Sigma_{i}\right)=A_{i}$. In this situation, following Gibbons [], we have a lower bound $A_{i}>16 \pi \mu^{2}$, or equivalently in terms of the area radius $R_{i}>2 \mu$. Indeed, letting

$$
\psi=\left(1+\frac{\mu}{2\left|x-x_{1}\right|}\right)
$$


then $\bar{h}=\psi^{4} \delta_{i j}$ is a Schwarzschild metric, $\bar{\Sigma}=\left\{\left|x-x_{1}\right|=\mu / 2\right\}$ minimizes area in $\bar{h}$ among all surfaces enclosing $E_{1}, \phi>\psi$, and hence:

$$
4 \pi R_{1}^{2}=A_{1}=A_{h}\left(\Sigma_{1}\right)>A_{\bar{h}}\left(\Sigma_{1}\right) \geq A(\bar{\Sigma})=16 \pi \mu^{2} .
$$

Similarly, $R_{2}>2 \mu$, and it follows immediately that $\frac{1}{2}\left(R_{1}+R_{2}\right)>2 \mu=m\left(E_{0}\right)$ violating (10).

The next proposition shows that for $\mu>0$ sufficiently small, there is no connected minimal sphere enclosing both $E_{1}$ and $E_{2}$, showing that for small values of $\mu>0$, the Brill-Lindquist data indeed provides a counterexample to (10).

Proposition 1. For sufficiently small $\mu>0$, the Brill-Lindquist initial data described above contain no closed connected minimal surface enclosing both punctures $x_{1}$ and $x_{2}$.

We remark that the statement follows from a direct application of Theorem 3.2 in [4. For the sake of completeness, we present the proof below. Our situation at hand is simpler than that of [4], and the proof below illustrates the geometry of the Brill-Lindquist metric concisely; see also [3] for a similar argument.

We also remark that this conclusion has been previously claimed based on numerical evidence presented in [2] and [6], and since then extensively numerically confirmed in the literature. Note that $\mu \rightarrow 0$ is equivalent to $L \rightarrow \infty$ while $\mu$ is kept constant, where $L$ is the separation distance between the punctures (with respect to the flat background metric). This limit can be interpreted as the Newtonian limit of the initial data. The physical content of this lemma is that in this limit the initial data correspond to two separated black holes.

Proof. To show the claim indeed holds, suppose $\Sigma$ is such a surface. Without loss of generality, we can assume that $\Sigma$ is the outermost minimal surface for the end $E_{0}$.

The configuration forces $\Sigma$ to intersect nontrivially with the $x y$-plane $\{z=$ $0\}$. Let $p$ be a point in $\Sigma \cap\{z=0\}$ and define the surface $\Sigma_{1}=\Sigma \cap B_{r}(p)$, with some fixed $r<1$. Here $B_{r}(p)$ denotes the (Euclidean) ball of radius $r$ centered at the point $p$. By construction, $\Sigma_{1}$ is a minimal surface, disjoint from the punctures, with nonempty boundary as the surface $\Sigma$ has to enclose both $x_{1}$ and $x_{2}$. Since, by assumption, $\Sigma$ is outermost, it is a stable minimal surface.

The following two consequences are then clearly in contradiction, implying that there is no such surface $\Sigma$.

i) By the Penrose inequality, the area of the surface $\Sigma$ (and hence the area of $\Sigma_{1}$ ) is bounded above by the total mass of the data (which in our configuration is given by $2 \mu$ );

$$
2 \mu \geq \sqrt{\frac{A(\Sigma)}{16 \pi}} \geq \sqrt{\frac{A\left(\Sigma_{1}\right)}{16 \pi}}
$$

And hence, we have that $A\left(\Sigma_{1}\right) \rightarrow 0$ as $\mu \rightarrow 0$. 
ii) We have a lower bound on the area $A\left(\Sigma_{1}\right)$ independent of $\mu$. This follows from an estimate of the sup norm of the second fundamental form as in Theorem 2 of [13] for stable minimal surfaces. It gives that the norm of the second fundamental form of $\Sigma_{1}$ is bounded uniformly (in $\mu$ ) by some positive constant $C$ (which depends on $0<r<1$ ), provided $\mu$ is sufficiently small. Then, there exists a sufficiently small $\varepsilon>0$ independent of $\mu$, so that the surface $\Sigma \cap B_{\varepsilon}(p)$ can be described as a graph over the tangent plane $T_{p} \Sigma$, which in turn gives a positive lower bound of the area of $\Sigma \cap B_{\varepsilon}(p)$ independent of $\mu$.

\section{Acknowledgments}

This work began at the conference "GR19, International Society on General Relativity \& Gravitation 19th International Conference", Mexico, July 5-9, 2010. S. D. and S. Y. would like to thank the organizers of this conference for the invitation.

S. D. is supported by CONICET (Argentina). This work was supported in part by grant PIP 6354/05 of CONICET (Argentina), grant 05/B415 SecytUNC (Argentina) and the Partner Group grant of the Max Planck Institute for Gravitational Physics, Albert-Einstein-Institute (Germany).

S.Y is partially supported by Grant-Aid for Scientific Research (No. 20540201).

\section{References}

[1] H. Bray. Proof of the Riemannian Penrose inequality using the positive mass theorem. J. Differential Geometry, 59:177-367, 2001.

[2] D. Brill and R. Lindquist. Interaction energy in geometrostatics. Phys. Rev., 131:471-476, 1963.

[3] P. T. Chruściel, J. Corvino and James Isenberg. Construction of $N$-body time-symmetric initial data sets in general relativity. arXiv:1004.1355, to appear in the Proceedings of Complex Analysis and Dynamical Systems IV.

[4] P. T. Chruściel and R. Mazzeo. On 'many-black-hole' vacuum spacetimes. Class. Quant. Grav., 20:729-754, 2003.

[5] S. Dain. Extreme throat initial data set and horizon area-angular momentum inequality for axisymmetric black holes. Phys. Rev. D, 82(10):104010, Nov 2010.

[6] G. Gibbons. The time symmetric initial value problem for black holes. Commun. Math. Phys., 27:87-102, 1972. 
[7] G. Gibbons. The isoperimetric and bogomolny inequalities for black holes. In T.J. Willmore and N. Hitchin, editors, Global Riemannian Geometry, pages 194-202. John Wiley \& Sons, New York, 1984.

[8] G. Huisken and T. Ilmanen. The inverse mean curvature flow and the Riemannian Penrose inequality. J. Differential Geometry, 59:352-437, 2001.

[9] P. Jang. Note on cosmic censorship. Phys. Rev. D, 20(4):834-837, 1979.

[10] M. Mars. Present status of the Penrose inequality. Class. Quant. Grav., 26:193001, 2009.

[11] W. Meeks III, L. Simon, and S. T. Yau. Embedded minimal surfaces, exotic spheres, and manifolds with positive Ricci curvature. Ann. of Math., 116(3):621-659, 1982.

[12] R. Penrose. Naked singularities. Ann. New York Acad. Sci., 224:125-134, 1973.

[13] R. Schoen. Estimates for stable minimal surfaces in three-dimensional manifolds. In Seminar on minimal submanifolds, volume 103 of Ann. of Math. Stud., pages 111-126. Princeton Univ. Press, Princeton, NJ, 1983.

[14] R. Schoen and S.-T. Yau. On the proof of the positive mass conjecture in general relativity. Comm. Math. Phys., 65(1):45-76, 1979.

[15] R. Schoen and S-T. Yau. Proof of the positive mass theorem. II. Comm. Math. Phys., 79(2):231-260, 1981.

[16] L. Szabados. Quasi-local energy-momentum and angular momentum in GR: A review article. Living Rev. Relativity, 7(4), 2004. cited on 8 August 2005.

[17] G. Weinstein and S. Yamada. On a Penrose inequality with charge. Commun. Math. Phys., 257(3):703-723, 2005.

[18] E. Witten. A new proof of the positive energy theorem. Communications in Mathematical Physics, 80:381-402, 1981. 10.1007/BF01208277. 